\title{
Stimulating a natural process for PFO closure
}

\author{
Thorsten Lewalter ${ }^{1,2}$ (DD
}

Received: 29 March 2019 / Accepted: 10 April 2019 / Published online: 27 April 2019

(C) Springer Science+Business Media, LLC, part of Springer Nature 2019

A patent foramen ovale can be found in around $25 \%$ of the population and is a risk factor for paradoxical embolism and stroke. After decades of controversial debates about the need for PFO closure by either surgical or transvenous intervention, it is now-after publication of several convincing prospective randomized trials [1-3]commonly accepted that in individuals with embolic stroke, no other discernable causes for stroke and a PFO with a significant inducible right-to-left shunting, PFO occlusion should be performed to minimize future risk of embolic stroke. The current clinical standard for PFO closure is the transvenous implantation of a septal occluding device. While the procedure is a relatively low-risk intervention, inter-atrial device implantation exhibitsaside from its beneficial effect on future stroke risk-a list of potential negative effects, caused by the device itself and its potential interaction with the surrounding cardiac structure: the occluding device may trigger atrial fibrillation and thus may induce the need for long-term oral anticoagulation! In addition, the surface of the occluder may serve as a source of embolism and thrombus formation; there is a small but possible risk of erosion of other cardiac structures such as the aorta or the roof of the left atrium. Furthermore, any future interventional catheter treatment which requires left atrial access has either to bypass the occluder or directly puncture and perforate the device itself, which therefore complicates any left atrial intervention, e.g., atrial fibrillation ablation or mitral valve clipping.

Therefore, the new idea presented by Di Biase and coworkers [4] is very important because it could serve as a new approach for PFO closure without requiring device

Thorsten Lewalter

th.lewalter@uni-bonn.de;

1 Department of Cardiology, Hospital Munich South, Munich, Germany

2 Univ. of Bonn, Bonn, Germany implantation - thus avoiding all of the abovementioned possible device-related complications.

In a first approach, ablation-induced PFO closure could be performed as a final treatment step in patients who already undergo ablation procedures for other reasons, when leaving the left atrium, in the sense of "closing the door when you leave the room." In line with the data that a PFO is a risk factor, this PFO closure would represent a primary preventative intervention and should reduce the future risk of such patient to experience paradoxical embolism. In a second step, such a device-free PFO closure could have the potential to partially or fully replace occluder implantation in a secondary or even primary preventative treatment strategy.

Data are presented from a first retrospective study as well as from a small prospective trial to support this device-free PFO closure concept. The authors adequately address all the obvious limitations, such as the small number of patients evaluated so far, or the chosen cardiac imaging modality. Therefore, there is a definite need to obtain more data for radiofrequency PFO closure, ideally as part of a larger prospective registry. Additionally, for this procedure, the currently best available imaging technique should be applied, which is a transesophageal echo, and the execution should be part of a standardized protocol which should include Valsalva maneuver.

The idea of using radiofrequency to create the adhesion of septum primum and septum secundum by stimulating a natural healing process is fascinating, offers several potential advantages over current closure techniques, and therefore urgently warrants further investigation!

\section{References}

1. Saver JL, Carroll JD, Thaler DE, for the RESPECT Investigators, et al. Long-term outcomes of patent foramen ovale closure or medical therapy after stroke. N Engl J Med. 2017;337:1030-2.

2. Mas JL, Derumeaux G, Guillon B, Massardier E, Hosseini H, Mechtouff L, et al. Patent foramen ovale closure or 
anticoagulation vs. antiplatelets after stroke. N Engl J Med. 2017;377:1011-21.

3. Søndergaard L, Kasner S, Rhodes JF, Andersen G, Iversen HK, Nielsen-Kudsk JE, et al. Patent foramen ovale closure or antiplatelet therapy for cryptogenic stroke. N Engl J Med. 2017;377:1033-42.
4. Di Biase L, et al. Closure of foramen ovale triggered by injury to tunnel surface of septum primum and secundum. JICE. 2019.

Publisher's note Springer Nature remains neutral with regard to jurisdictional claims in published maps and institutional affiliations. 